\title{
The Role of Nitrogen in Defect Evolution in Zinc Oxide: STEM-EELS Nanoscale Investigations
}

\author{
Calliope Bazioti, Alexander Azarov*, Klaus M. Johansen, \\ Bengt G. Svensson, Lasse Vines, Andrej Y. Kuznetsov, Øystein Prytz \\ Department of Physics, Centre for Materials Science and Nanotechnology, \\ University of Oslo, P.O. Box 1048, Blindern, N-0316 Oslo, Norway
}

\begin{abstract}
Direct evidence of nitrogen-molecule $\left(\mathrm{N}_{2}\right)$ formation after ion-implantion of $\mathrm{ZnO}$ has been found in an atomically-resolved STEM-EELS investigation. Taking advantage of the possibility to use multiple detectors simultaneously in aberration-corrected STEM, the detailed correlation between atomic structure and chemical identification is utilized to develop a model explaining the formation and evolution of different defect-types and their interaction with $\mathrm{N}$. In particular, the formation of zinc vacancy $\left(\mathrm{V}_{\mathrm{Zn}}\right)$-clusters filled with $\mathrm{N}_{2}$ after heat treatment at $650^{\circ} \mathrm{C}$ was observed, clearly indicating that $\mathrm{N}$ has not been stabilized in the $\mathrm{O}$ substitutional site, thus limiting p-type doping. Previous results, showing an exceptional thermal stability of vacancy-clusters only for the case of $\mathrm{N}$-doped $\mathrm{ZnO}$ are supported. Furthermore, the $\mathrm{V}_{\mathrm{Zn}}-\mathrm{N}_{2}$ stabilization leads to suppression of $\mathrm{V}_{\mathrm{Zn}}-\mathrm{Zn}_{\mathrm{i}}$ recombination, hence the highly mobile $\mathrm{Zn}$ interstitials preferentially condense on the basal planes promoting formation of extended defects (basal stacking faults, stacking mismatched boundaries). The terminations of these defects provide energetically favourable sites for further $\mathrm{N}_{2}$-trapping as a way to reduce local strain fields.
\end{abstract}

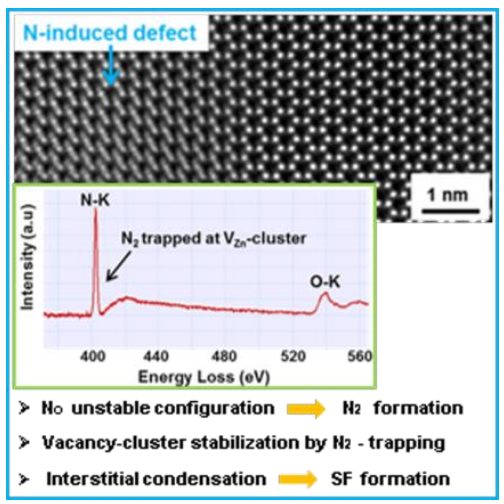

* NOMATEN CoE, National Centre for Nuclear Research, A. Soltana 7, 05-400 Otwock-Świerk, Poland 
Point and extended defects, as well as their interaction with dopants, directly influence semiconductor properties hence, identifying different defect-types and understanding their formation and evolution is crucial for mastering semiconductors. The prominent correlation between defects and electrical-optical properties is clearly evident in the case of $\mathrm{ZnO}$, a wide and direct band-gap semiconductor $(\sim 3.4 \mathrm{eV})$ with a potential for a variety of next generation solid-state devices in the fields of optoelectronics, quantum technology, spintronics and radiation sensors in harsh environments ${ }^{1,2,3}$. However, the doping asymmetry, where only n-type conductivity can be readily accomplished, hinders the realization of $\mathrm{ZnO}$ devices ${ }^{4,5}$. Nitrogen $(\mathrm{N})$, has been considered as one of the most promising candidates for p-type doping in $\mathrm{ZnO}$, resulting in intensive studies regarding $\mathrm{N}$-behavior in $\mathrm{ZnO}^{6,7,8,9,10,11}$. However, the results obtained so far are quite contradictory and reliable p-type doping is still challenging. $\mathrm{N}$-doped $\mathrm{ZnO}$ has also attracted fundamental interest since it exhibits a remarkable behavior in terms of defect evolution, in comparison to other dopants. In particular, Børseth et al. ${ }^{12}$ and Tuomisto et al. ${ }^{13}$ revealed by positron annihilation spectroscopy (PAS) an exceptional stability of $\mathrm{Zn}$ vacancy $\left(\mathrm{V}_{\mathrm{zn}}\right)$-clusters for $\mathrm{N}$ doped $\mathrm{ZnO}$. This observation was irrespective of the growth/implantation methods, revealing the generality of the phenomenon. Furthermore, Azarov et al. ${ }^{14,15}$ showed by ion channeling measurements that defects induced by $\mathrm{N}$-implantation into $\mathrm{ZnO}$ ( $\mathrm{Zn}$ interstitial-type defects) exhibit nonlinear thermal evolution trends, demonstrating a dramatic increase in the defect concentration occurring at $650^{\circ} \mathrm{C}$ (so-called reverse annealing). The exact mechanism for reverse annealing is not yet understood, but the effect occurs exclusively for the $\mathrm{N}$ implants. Hence, it is evident that the defect formation/evolution in $\mathrm{ZnO}$ hinges strongly on the implanted species $^{12,13,14,16,17}$, and detailed nano-structural and chemical defect characterization is of utmost importance to reveal the role of $\mathrm{N}$ in the complex defect interplay.

Scanning Transmission Electron Microscopy combined with Electron Energy-Loss Spectroscopy (STEMEELS) is a unique tool for both imaging and spectroscopy on the atomic scale, giving invaluable insight into the defect interaction and evolution. In the present work, an atomically-resolved STEM-EELS study was performed for the first time on $\mathrm{N}$-implanted wurtzite $\mathrm{ZnO}$ single crystals that were heat-treated at $650^{\circ} \mathrm{C}$, in order to elucidate the character and interaction of $\mathrm{N}$-related defects. As a result, we propose a model that describes the defect interplay, starting from point defects behavior (interstitials $\left(\mathrm{Zn}_{\mathrm{i}}, \mathrm{O}_{\mathrm{i}}\right)$ and vacancies $\left(\mathrm{V}_{\mathrm{zn}}, \mathrm{V}_{\mathrm{o}}\right)$ ) promoting the formation of extended defects (V-clusters, stacking faults), and revealing the decisive role of $\mathrm{N}$ in defect evolution. The correlation between atomic structure and chemical information on the atomic scale was crucial for the apparent study, hence we took advantage of the possibility to use multiple detectors, in order to obtain simultaneous information through different mechanisms of the electron beam-specimen interactions. In this respect, we performed: a) Simultaneous acquisition of annular bright field (ABF), annular dark field (ADF) and high-angle annular dark field (HAADF) images, providing complementary views of the same region. In particular, $\mathrm{ABF}$ is a direct method of visualizing light and heavy elements simultaneously, ADF provides strong diffraction contrast while in HAADF contrast is directly correlated to the atomic number (Z-contrast). b) Simultaneous STEM imaging and EELS on the atomic scale, allowing for correlation between atomic structure and chemical information. Geometric Phase Analysis (GPA) was performed on high resolution STEM images to extract lattice phase maps and for the nanoscale localization of strained regions.

Figure 1a presents a low-magnification ADF-STEM image with an overlay of the N-implanted profile calculated by the Transport of Ions in Matter (TRIM) code ${ }^{18}$. The diffraction contrast of the ADF image reveals that the average depth of the implanted zone is close to $120 \mathrm{~nm}$ from the surface, which is in agreement with the TRIM calculations and the maximum ion concentration is expected approximately at $\sim 80 \mathrm{~nm}$ from the surface. Figures $1 \mathrm{~b}$ and 1c present high resolution ADF-STEM and HAADF-STEM images respectively, that were recorded simultaneously, illustrating the defect formation in the area close to the maximum ion concentration. 
The ADF image provides strong diffraction contrast, hence the localization of extended defects is easily detectable. In addition to the extended defects, at the corresponding HAADF image we observe the formation of nano-sized areas exhibiting darker contrast. Accounting that HAADF is a pure Z-contrast image, formed by scattering from heavy elements (in our case $\mathrm{Zn}$ ), these dark nano-sized areas can be indicative of volumes with lower atomic density (e.g. vacancy-clusters) or/and clustering of light elements.
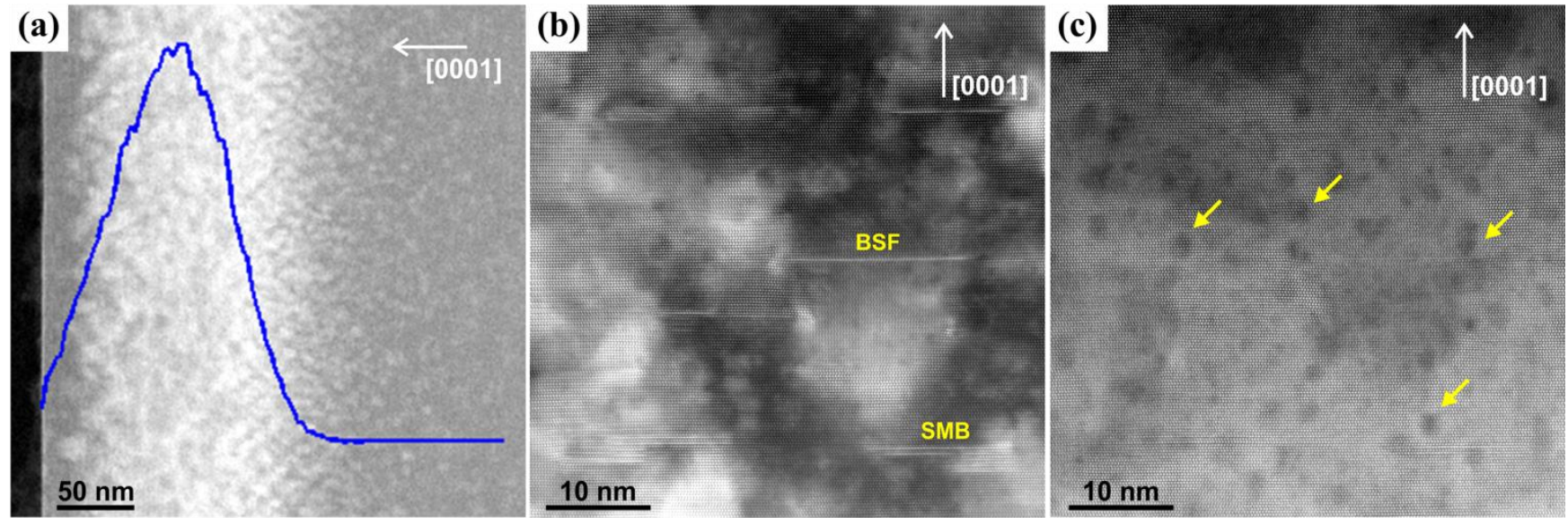

Figure 1. (a) Low-magnification ADF-STEM image with an overlay of the N-implanted profile calculated by the TRIM code. Both imaging and TRIM data are in agreement revealing an implanted area of $\sim 120 \mathrm{~nm}$, with the maximum ion concentration at $\sim 80 \mathrm{~nm}$ from the surface. (b) ADF-STEM and (c) HAADF-STEM images recorded simultaneously, close to the maximum ion concentration region. Extended defects are easily detectable in the ADF image due to strong diffraction contrast while the HAADF Z-contrast image additionaly reveals the existence of nano-sized cluster-like defects with lower atomic density.

\section{(i) Extended defects}

Formation of basal stacking faults (BSFs) was found to dominate the N-implanted region. The wurtzite (WZ) structure is described by the stacking of close-packed double layers of (0001) planes (basal planes) and the perfect stacking sequence along the [0001] direction is $\mathrm{ABABAB}$, where each letter corresponds to a $\mathrm{Zn}-\mathrm{O}$ bilayer. Figures $2 \mathrm{a}$ and $2 \mathrm{~b}$ show an atomically-resolved $\mathrm{ABF}$ image recorded from the middle part of a BSF, allowing for simultaneous direct visualization of heavy and light elements. The $\mathrm{Zn}-\mathrm{O}$ dumbbells are resolved and a BSF of $\mathrm{I}_{1}$-type was identified, characterized by one violation of the stacking rule, changing the stacking sequence according to $\mathrm{ABABCBCB}$. These stacking faults introduce one cubic structural unit in the stacking sequence (Figure 2c). Their average lateral extension after the annealing process is up to $20 \mathrm{~nm}$ and they can be formed due to agglomeration of self-interstitial atoms $\left(\mathrm{Zn}_{\mathrm{i}}, \mathrm{O}_{\mathrm{i}}\right)$ into the basal planes, since they were found to be of interstitial-type due to the insertion of an extra half-plane (Figures 2d-2e). They are usually bounded by Frank-Shockley dislocations with Burgers vectors $b=1 / 6\langle 02 \overline{2} 3\rangle=1 / 2\langle 0001\rangle+1 / 3\langle 01 \overline{1} 0\rangle$ (components) ${ }^{19}$. The $1 / 3\langle 0001\rangle$ Burgers vector component alters the stacking sequence of the perfect $\mathrm{WZ}$ crystal, while the $1 / 3\langle 01 \overline{1} 0\rangle$ causes a slip of the upper part of the $\mathrm{ZnO}$ crystal with respect to the lower part as we cross the BSF (Figures $2 \mathrm{f}-2 \mathrm{~g})^{20}$. Note that basal stacking faults are the most common ones in wurtzite materials, and Wang et al. ${ }^{21}$ have also suggested that interstitials condense on the basal plane. 

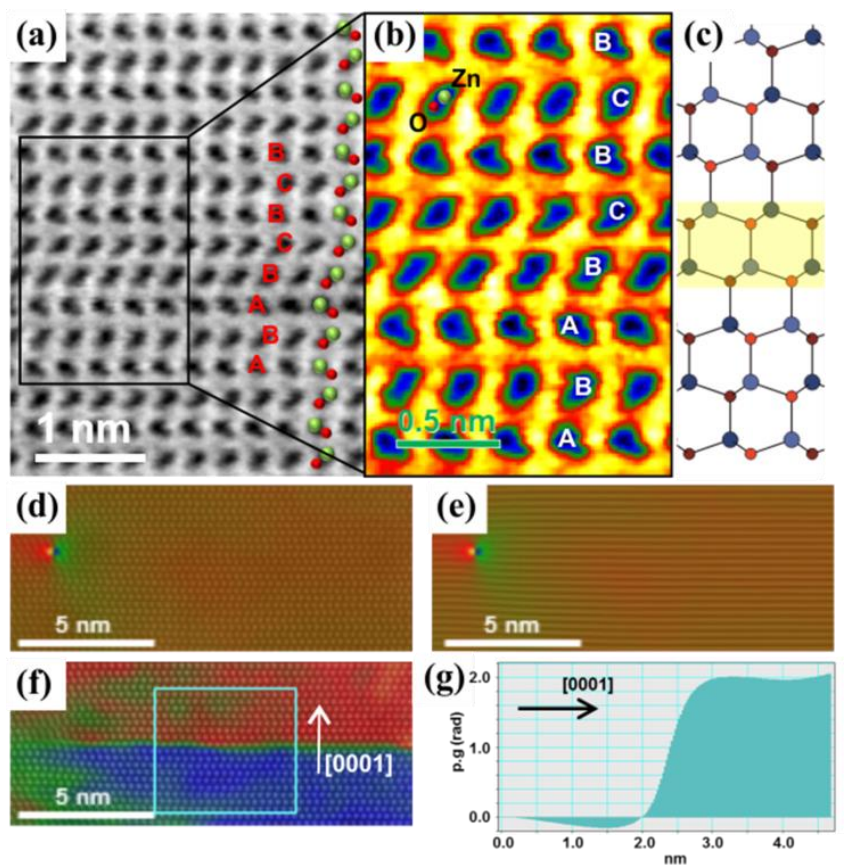

Figure 2. (a) High-resolution ABF-STEM image of a BSF of $I_{1}$-type and (b) the corresponding enlarged colored image. The $\mathrm{Zn}-\mathrm{O}$ dumbbells are easily resolved, revealing a change in the stacking sequence according to ABABCBCB. (c) Structural model of the $I_{1}$-BSFs showing the insertion of one cubic layer in the wurtzite structure. (d) Overlap of HAADF image and the corresponding $\varepsilon_{y y}$ strain map, acquired from the left termination of the BSF, (e) the corresponding overlap of the $\varepsilon_{y y}$ strain map and Bragg filtering image with g0002. The insertion of an extra half plane is illustrated, indicating that the BSF is of interstitial-type. (f) Overlap of HAADF image and the $\mathrm{g} 1 \overline{1} 00$ phase map and $(\mathrm{g})$ the corresponding phase profile showing a phase change $\sim 2 \mathrm{rad}$ across the $\mathrm{I}_{1}$-BSF, consistent with the in-plane shift of the crystal equal to $1 / 3$ [11 100$]$.

Extrinsic basal stacking faults (E-BSFs) exhibiting smaller lateral extension $(\sim 5 \mathrm{~nm})$ were also observed. The E-BSF is described by a stacking sequence according to ABABCABAB (Figures $3 \mathrm{a}$ and $3 \mathrm{~b}$ ) and introduces three cubic structural units in the wurtzite structure (Figure 3c). Similar to the $\mathrm{I}_{1}$-BSFs, they are formed by the precipitation of self-interstitial atoms $\left(\mathrm{Zn}_{\mathrm{i}}, \mathrm{O}_{\mathrm{i}}\right)$ on the basal plane, since they were also found to be of interstitialtype due to insertion of extra half-planes (Figures $3 \mathrm{~d}$ and 3e). They are bounded by Frank dislocations with Burgers vectors $b=1 / 2\langle 0001\rangle$, so no slip is caused of the upper part of the $\mathrm{ZnO}$ crystal with respect to the lower part (Figures $3 \mathrm{f}$ and $3 \mathrm{~g})^{20}$. However, these faults require higher energy for their formation than $\mathrm{I}_{1}$-BSFs, taking into account that the formation energy is almost proportional to the number of the inserted cubic layers ${ }^{22}$. This justifies the fact that we always observe them in smaller lateral extensions. 

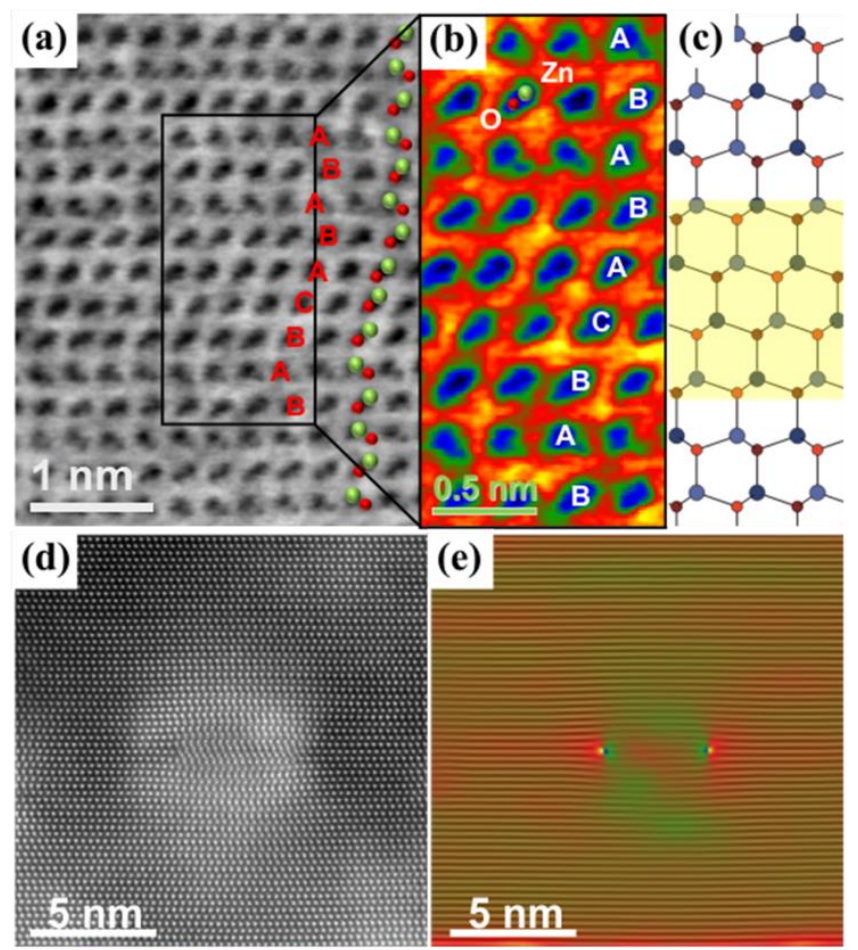

(f)
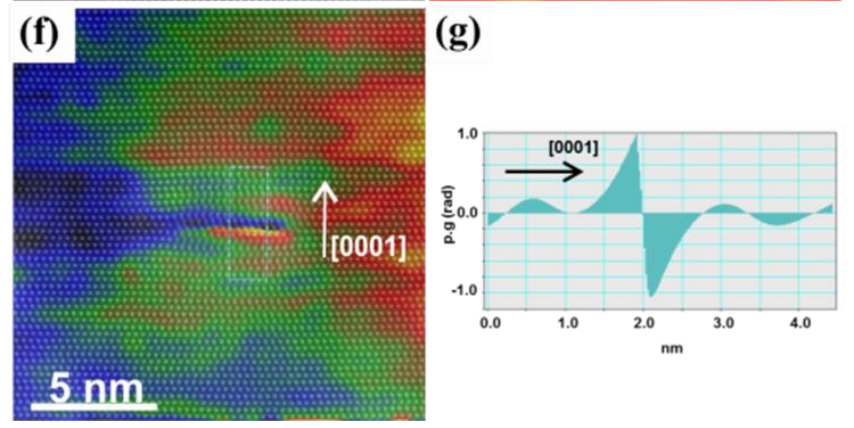

Figure 3. (a) High-resolution ABF-STEM image of a BSF of E-type and (b) the corresponding enlarged colored image. The $\mathrm{Zn}-\mathrm{O}$ dumbbells are easily resolved, revealing a change in the stacking sequence according to ABABCABAB. (c) Structural model of the E-BSFs showing the insertion of three cubic layers in the wurtzite structure. (d) High resolution ADF image and (e) the corresponding $\varepsilon_{\mathrm{yy}}$ strain map overlapped with the Bragg filtering image with g0002. The insertion of extra half-planes is illustrated, indicating the interstitial nature of the defect. (f) Overlap of HAADF image and g1 100 phase map and (g) the corresponding phase profile showing no phase change across the E-BSF, indicating zero in-plane shift.

Another extended defect-type observed is the stacking mismatched boundary (SMB), characterized by the 'double' positioned monolayers of Zn (Figure 4). SMBs are prismatic stacking faults (PSFs) on the (1210) plane (proposed by Amelinckx) ${ }^{23}$ and they exhibit an average lateral extension close to $20 \mathrm{~nm}$. SMBs act as connections between two $\mathrm{I}_{1}$-BSFs lying on different basal planes (also shown by Knoll et al. ${ }^{24}$ ). The $\mathrm{I}_{1}$-type BSFs frequently appear in pairs separated by 2-3 unit cells along the c-axis after which the original stacking sequence is restored. This arrangement is energetically favorable, as the strain fields at one side of each BSF can overlap and cancel. 

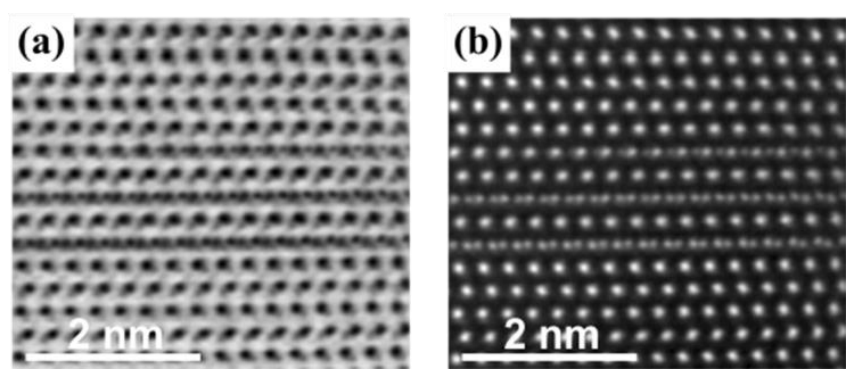

Figure 4. High-resolution STEM images of a stacking mismatched boundary formed by a prismatic stacking fault on the (12 10$)$ plane. In the ABF image on the left (a) the $\mathrm{Zn}-\mathrm{O}$ dumbbells are resolved, while the HAADF image on the right (b) shows the position of the $\mathrm{Zn}$ atomic columns.

\section{(ii) V-cluster stabilization by $\mathbf{N}_{2}$}

In addition to the extended defects (Figure 1b), the corresponding HAADF image (Figure 1c) reveals the formation of nano-sized areas exhibiting darker contrast. This is indicative of reduced atomic mass density, indicating that vacancy clusters or/and clustering of light elements are formed.

In order to investigate this, atomic scale STEM-EELS was performed on these regions. Figure 5a shows an ADF-STEM intensity map across a nano-sized dark area, close to the maximum ion concentration region. The corresponding relative thickness map shows a clear reduction in the effective thickness (Figure 5b) correlated to a local Zn-depletion, indicating the excistence of a nano-sized $\mathrm{V}_{\mathrm{Zn}}$-cluster. In addition, the corresponding N-K intensity EELS map (Figure 5c) reveals a N-rich region. The EELS spectra (Figure 5d) show that (1) nitrogen is localized exactly inside the $\mathrm{V}_{\mathrm{Zn}}$-cluster, while in the surrounding area the $\mathrm{N}-\mathrm{K}$ signal disappears abruptly, indicating an almost pure $\mathrm{ZnO}$ 'matrix'. Moreover, the shape and the energy position of the N-K edge indicate the formation of molecular nitrogen $\left(\mathrm{N}_{2}\right)$, -as shown in Figure 5e, where spectra from monochromated STEMEELS are compared to reference spectra of gaseous $\mathrm{N}_{2}$ and $\mathrm{GaN}$-. Hence, the formation of $\mathrm{N}_{2}$-filled nano-voids $\left(\mathrm{V}_{\mathrm{Zn}}-\mathrm{N}_{2}\right.$ clusters) is identified. In general, they appear to be randomly distributed in the implanted region however, a more careful inspection reveals that they are typically formed in the vicinity of the extended defects. Figure $5 \mathrm{f}$ reveals the formation of a void at the termination of a basal stacking fault. In particular, the cores of partial edge dislocations that bound the BSFs can act as strong sinks for dopants, accommodating local strain fields (Figures $5 \mathrm{~g}$-i). 

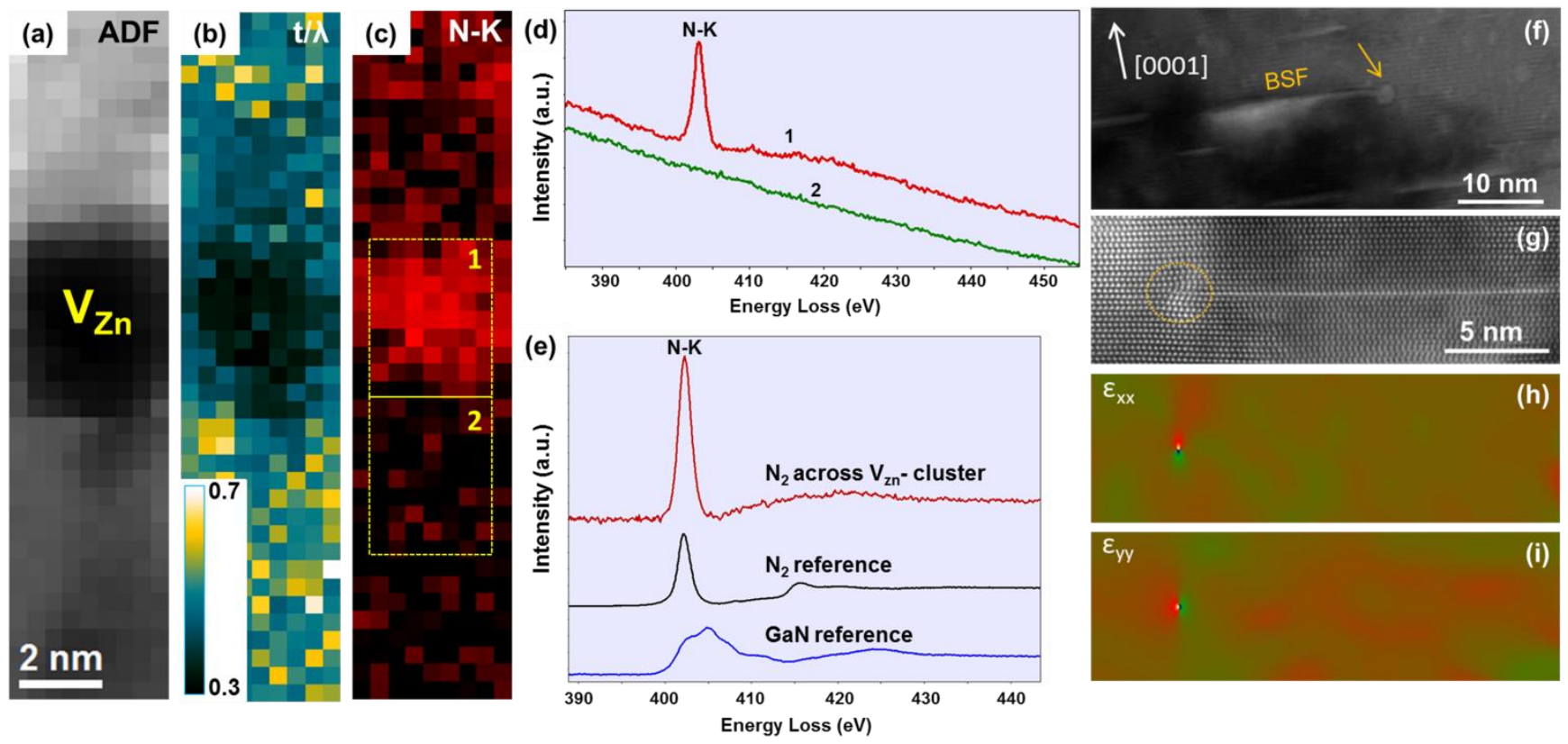

Figure 5. (a) ADF-STEM intensity map across a nano-void and the corresponding (b) relative thickness map and (c) N-K intensity EELS map. A clear drop in ADF intensity and thickness are indicative of a Zn-depleted area $\left(\mathrm{V}_{\mathrm{Zn}}\right.$ - cluster). (d) The corresponding EELS-spectra acquired from the area (1) inside and (2) outside the $\mathrm{V}_{\mathrm{Zn}}$ - cluster. $\mathrm{N}-\mathrm{K}$ signal is detected only inside the $\mathrm{V}_{\mathrm{Zn}}$ - cluster and disappears abruptly outside it. (e) The shapes of the $\mathrm{N}-\mathrm{K}$ peaks are clearly illustrated, where monochromated spectra from: our experiment from the Nimplanted $\mathrm{ZnO}$ sample, $\mathrm{N}_{2}$ reference ${ }^{25}$ and our experiment from a $\mathrm{GaN}$ reference sample are compared. Hence, the formation of a $\mathrm{V}_{\mathrm{Zn}}-\mathrm{N}_{2}$ cluster surrounded by an almost pure $\mathrm{ZnO}$ matrix is identified. (f) Bright field TEM image under two-beam conditions with g1 100 revealing a nano-cluster localization at the termination of a basal stacking fault, (g)-(i) ABF-STEM image and the corresponding GPA strain maps, showing local strain fields associated with the partial edge dislocations bounding the BSFs. The cores of dislocations can act as strong sinks of dopants accommodating the local strain fields.

Our experimental STEM-EELS results are in agreement with previous theoretical/experimental studies and go further in terms of the comprehensive nanostructural picture regarding the N-behavior in $\mathrm{ZnO}$. According to calculations of Nickel and $\mathrm{Gluba}^{26}$, the $\mathrm{O}$ site is energetically unfavorable for the localization of $\mathrm{N}_{2}$. Furthermore, first-principles calculations by Lambrecht and Boonchun ${ }^{27}$ showed that $\mathrm{N}_{2}$ may occupy a $\mathrm{V}_{\mathrm{Zn}}$. Photo-Electron Paramagnetic Resonance (EPR) measurements by Philipps et al. ${ }^{28}$ indicated that the $\mathrm{N}_{2}$ acceptor level is even deeper than $\mathrm{N}_{\mathrm{O}}$ and $\mathrm{N}_{2}$ may exist as a molecule in interstitial locations or voids. Soudi et al. ${ }^{29}$ observed N-related complexes in MBE-grown $\mathrm{ZnO}$ nanowires performing Raman spectroscopy. $\mathrm{N}_{2}$ molecule was observed by X-ray absorption experiments in the annealing process of MBE-grown $\mathrm{ZnO}: \mathrm{N}$ by Fons et al. ${ }^{30}$, proposing that annealing caused the formation of $\mathrm{N}_{2}$ bubbles.

In order to understand the formation mechanism of $\mathrm{N}_{2}$ in the $\mathrm{N}$-implanted samples, it is worth notting that the nitrogen is introduced in the form of ionized $\mathrm{N}^{+}$and after implantation $\mathrm{N}$ is expected to be placed either in an interstitial position $\left(\mathrm{N}_{\mathrm{i}}\right)$ or substitutional position $\left(\mathrm{N}_{\mathrm{O}}\right)$ in the oxygen sub-lattice. However, according to our STEM-EELS results, signals revealing $\mathrm{Zn}-\mathrm{N}$ bonds were not observed. This indicates that $\mathrm{N}$ taking a substitutional $\mathrm{O}$ position $\left(\mathrm{N}_{\mathrm{O}}\right)$ is not a stable configuration. It has been previously reported that the low p-type 
doping efficiency of $\mathrm{N}$ in $\mathrm{ZnO}$ could be attributed to the instability of $\mathrm{N}_{\mathrm{O}}$ due to the unfavorable local chemical environment (Fons et al. ${ }^{30}$ ). DFT calculations by Gao et al. ${ }^{31}$ indicated that the existence of $\mathrm{O}_{i}$ plays a decisive role in $\mathrm{N}_{\mathrm{O}}$ deactivation and formation of $\mathrm{N}_{2}$. In particular, $\mathrm{N}_{\mathrm{O}}$ would be easily kicked out to an interstitial site $\left(\mathrm{N}_{\mathrm{i}}\right)$ by $\mathrm{O}_{\mathrm{i}}$, due to their attractive interaction. Furthermore, their calculations showed that the $\mathrm{N}_{\mathrm{i}}-\mathrm{N}_{\mathrm{i}}$ binding energy is large due to the large strength of the $\mathrm{N} \equiv \mathrm{N}$ triple bond, hence the total energy drops dramatically once two $\mathrm{N}_{\mathrm{i}}$ bind together to form $\mathrm{N}_{2}$.

Furthermore, our STEM-EELS investigation revealing localization of $\mathrm{N}_{2}$ at $\mathrm{V}_{\mathrm{Zn}}$-clusters, explains results previously reported by Børseth et al. ${ }^{12}$ and Tuomisto et al. ${ }^{13}$. In particular, they detected by PAS the formation of $\mathrm{V}_{\mathrm{zn}}$-clusters in $\mathrm{N}$-doped $\mathrm{ZnO}$. These vacancy-clusters with an open volume larger than that of a $\mathrm{Zn}$ monovacancy already exist in the as-implanted $\mathrm{ZnO}$ and after heat treatment at $600^{\circ} \mathrm{C}$ they start to grow in size. Interestingly, they showed that $\mathrm{V}_{\mathrm{zn}}$-clusters exhibit an exceptional thermal stability even at higher temperatures, which is not typically observed for other dopants. According to our results, the thermal stability could be associated with the localization of $\mathrm{N}_{2}$ molecules in the $\mathrm{V}_{\mathrm{zn}}$-clusters, possibly reducing strain due to local lattice distortion. It is worth noting that with PAS it is not possible to directly detect $\mathrm{V}_{\mathrm{O}}$, but it is highly likely that the $\mathrm{V}$-clusters observed by PAS and STEM are also a combination of both $\mathrm{V}_{\mathrm{Zn}}$ and $\mathrm{V}_{\mathrm{O}}$.

Our results, showing $\mathrm{N}_{2}$-filled $\mathrm{V}_{\mathrm{zn}}$-clusters, also explain the formation of the extended basal stacking faults. The stabilization of $\mathrm{V}_{\mathrm{zn}}$-clusters by $\mathrm{N}_{2}$ suppresses the $\mathrm{V}_{\mathrm{zn}}-\mathrm{Zn}_{\mathrm{i}}$ recombination, which results initially in a high $\mathrm{Zn}_{\mathrm{i}}$ concentration in the implanted region. The resulting local strain in addition to the high $\mathrm{Zn}_{\mathrm{i}}$ mobility even at room temperature can easily lead to migration of $\mathrm{Zn}_{\mathrm{i}}$, that preferentially condense on the (0001) basal planes, resulting in formation of interstitial BSFs (insertion of extra planes). It is essential to emphasize that the BSFs formation needs the precipitation of a complete $\mathrm{Zn}-\mathrm{O}$ bilayer, meaning that in addition to agglomeration of $\mathrm{Zn}_{\mathrm{i}}$, also $\mathrm{O}_{\mathrm{i}}$ should diffuse at the same time. Further diffusion of interstitial point defects into the basal plane gradually increases the BSF size leading to a partial strain saturation.

Regarding the formation energies of BSFs in $\mathrm{ZnO}$, calculations by Yanfa Yan et al. ${ }^{22}$ showed that in general all BSFs exhibit low formation energies, -comparable to what is observed in GaN-. This indicates that they can form easily through kinetics in $\mathrm{ZnO}$. The BSFs of $\mathrm{I}_{1}$-type that are dominant in our case, are energetically favorable compared to other BSF-types. E-BSFs are less energetically favorable, justifying the fact that we always observe them in smaller lateral extensions. As the E-BSFs grow in size, the excess stacking fault energy can only be partially reduced by changing the loop structure from E-type to $\mathrm{I}_{1}$-type. This can happen by the nucleation of a partial dislocation $b=1 / 3\langle 01 \overline{1} 0\rangle$ that shears the E-BSF into $\mathrm{I}_{1}$-BSF (dissociation of Frank loop into Frank-Shockley) ${ }^{32}$. This implies that part of the $\mathrm{I}_{1}$-BSFs that we observe can be the result of E-BSFs dissociation.

Finally, the terminations of the extended defects that we observed (BSFs and SMBs), can provide energetically favorable sites for further $\mathrm{N}_{2}$ localization. In particular, the cores of partial edge dislocations that bound the SFs can act as strong sinks of dopants, accommodating the local strain fields ${ }^{33}$. 


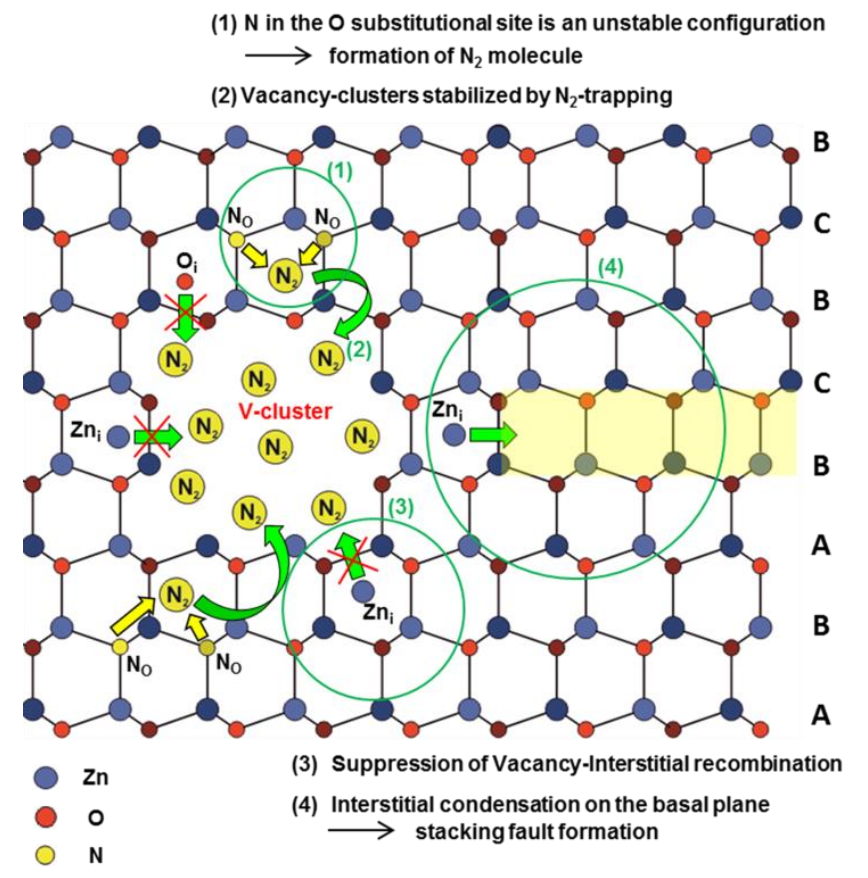

Figure 6. Model describing defect evolution in $\mathrm{N}$-implanted $\mathrm{ZnO}$.

In conclusion, through a comprehensive nanostructural and chemical investigation we have elucidated the behavior of nitrogen into a $\mathrm{ZnO}$ host matrix and described a model regarding the defect evolution (Figure 6). Our EELS results show direct evidence for the formation of $\mathrm{N}_{2}$ molecules, thereby supporting previous reports on the low stability of $\mathrm{N}$ subsitution on $\mathrm{O}$ sites. Furthermore, we propose that $\mathrm{V}_{\mathrm{Zn}}$-clusters are stabilized by the presence of $\mathrm{N}_{2}$, resulting in an exceptional thermal stability compared to the situation with other dopants, and leading to a suppression of $\mathrm{V}_{\mathrm{zn}}-\mathrm{Zn}_{\mathrm{i}}$ recombination. Thus the highly mobile $\mathrm{Zn}$ interstitials can easily migrate and form interstitial-type extended defects. In particular, $\mathrm{Zn}_{\mathrm{i}}$ preferentially condenses on the basal planes, resulting in the formation of basal stacking faults that grow in size leading to strain saturation. The dominant BSF-type is the energetically favorable $\mathrm{I}_{1}$-type terminated by stacking mismatched boundaries or Frank-Shockley dislocations, which again provide energetically favorable sites for further $\mathrm{N}_{2}$-trapping as a way to reduce local strain fields.

\section{Corresponding author}

kalliopi.bazioti@smn.uio.no

\section{Supporting Information Available:}

Experimental methods employed; Ion-implantation process, Monte Carlo simulations using the TRIM code, (Scanning) Transmission Electron Microscopy ((S)TEM), Electron Energy-Loss Spectroscopy (EELS), Geometric Phase Analysis (GPA), TEM sample preparation, sample thickness evaluation by low-loss EELS. 


\section{Acknowledgments}

The Research Council of Norway is acknowledged for the support to the Norwegian Center for Transmission Electron Microscopy (NORTEM) (no. 197405/F50), the Norwegian Micro and Nano-Fabrication Facility (NorFab) (no. 245963/F50) and the top-tier research project FUNDAMeNT (no. 251131).

\section{References}

(1) Djurišić, A. B.; Ng, A. M. C.; Chen, X. Y. ZnO Nanostructures for Optoelectronics: Material Properties and Device Applications. Prog. Quantum Electron 2010, 34, 191-259.

(2) Özgür, Ü.; Alivov, Y. I.; Liu, C.; Teke, A.; Reshchikov, M. A.; Doğan, S.; Avrutin, V; Cho, S.-J.; Morkoç, H. A Comprehensive Review of ZnO Materials and Devices. J. Appl. Phys. 2005, 98, 041301.

(3) Liu, C.; Yun, F.; Morkoç H. Ferromagnetism of ZnO and GaN: A Review. J. Mater. Sci.: Mater. Electron 2005, 16, 555-597.

(4) Avrutin, V.; Silversmith, D. J.; Morkoç, H. Doping Asymmetry Problem in ZnO: Current Status and Outlook. Proc. IEEE 2010, 98, 1269-1280.

(5) Vines, L.; Kuznetsov, A. Bulk Growth and Impurities. Semiconductors and Semimetals 2013, 88, 67-104.

(6) Lautenschlaeger, S.; Eisermann, S.; Haas, G.; Zolnowski, E. A.; Hofmann, M. N.; Laufer, A.; Pinnisch, M.; Meyer, B. K.; Wagner, M. R.; Reparaz, J. S. et al. Optical Signatures of Nitrogen Acceptors in ZnO. Phys. Rev. B 2012, 85, 235204.

(7) Myers, M. A.; Myers, M. T.; General, M. J.; Lee, J. H.; Shao, L.; Wang, H. P-type ZnO Thin Films Achieved by N ${ }^{+}$Ion Implantation through Dynamic Annealing Process. Appl. Phys. Lett. 2012, 101, 112101.

(8) Ding, M.; Zhao, D.; Yao, B.; Li, B.; Zhang, Z.; Shen, D. The P-Type ZnO Film Realized by a Hydrothermal Treatment Method. Appl. Phys. Lett. 2011, 98, 062102.

(9) Kennedy, J.; Carder, D. A.; Markwitz, A.; Reeves, R. J. Properties of Nitrogen Implanted and Electron Beam Annealed Bulk ZnO. J. Appl. Phys. 2010, 107, 103518.

(10) Reynolds, J. G.; Reynolds, C. L.; Mohanta, Jr. A.; Muth, J. F.; Rowe, J. E.; Everitt, H. O.; Aspnes, D. E. Shallow Acceptor Complexes in P-Type ZnO. Appl. Phys. Lett. 2013, 102, 152114.

(11) Barnes, T. M.; Olson, K.; Wolden, C. A. On the Formation and Stability of P-Type Conductivity in Nitrogen-Doped Zinc Oxide. Appl. Phys. Lett. 2005, 86, 112112.

(12) Børseth, T. M.; Tuomisto, F.; Christensen, J. S.; Monakhov, E. V.; Svensson, B. G.; Kuznetsov, A. Yu. Vacancy Clustering and Acceptor Activation in Nitrogen-Implanted ZnO. Phys. Rev. B 2008, 77, 045204.

(13) Tuomisto, F.; Rauch, C.; Wagner, M. R.; Hoffmann, A.; Eisermann, S.; Meyer, B. K.; Kilanski, L.; Tarun, M. C.; McCluskey, M. D. Nitrogen and Vacancy Clusters in ZnO. J. Mater. Res. 2013, 28, 1977-1983.

(14) Azarov, A.; Galeckas, A.; Wendler, E.; Ellingsen, J.; Monakhov, E.; Svensson, B. G. Normal and Reverse Defect Annealing in Ion Implanted II-VI Oxide Semiconductors. J. Appl. Phys. 2017, 122, 115701.

(15) Azarov, A.Y.; Halen, A.; Du, X. L.; Rauwel, P.; Kuznetsov, A. Y.; Svensson, B. G. Effect of Implanted Species on Thermal Evolution of Ion-Induced Defects in ZnO. J. Appl. Phys. 2014, 115, 073512.

(16) Chen, Z. Q.; Maekawa, M.; Kawasuso, A.; Suzuki, R.; Ohdaira, T. Interaction of Nitrogen with Vacancy Defects in $\mathrm{N}^{+}-$ Implanted ZnO Studied Using a Slow Positron Beam. Appl. Phys. Lett. 2005, 87, 091910.

(17) Sonder, E.; Zhur, R. A.; Valiga, R. E. Annealing of Damage and Stability of Implanted Ions in ZnO Crystals. J. Appl. Phys. 1988, 64, 1140-1144.

(18) Ziegler, J. F.; Biersack, J. P.; Littmark, U. The Stopping and Range of Ions in Solids; Pergamon Press: New York, 1985.

(19) Liliental-Weber, Z. Structural Defects in GaN Revealed by Transmission Electron Microscopy, Jpn. J. Appl. Phys. 2014, 53, 100205.

(20) The phase is defined as $P_{g}=-2 \pi \mathbf{g} . \mathbf{u}$, where $\mathbf{g}$ is the reciprocal lattice vector and $\mathbf{u}$ is the lattice displacement. This results in $\mathrm{P}_{\mathrm{g}}=-2 \pi\left(\mathrm{u}_{\mathrm{g}} / \mathrm{d}_{\mathrm{g}}\right)$ where $\mathrm{u}_{\mathrm{g}}$ is the magnitude of the projected displacement along the $\mathrm{g}$ vector and $\mathrm{d}_{\mathrm{g}}$ the corresponding lattice spacing.

(21) Wang, C. M.; Jiang, W.; Weber, W. J.; Thomas, L. E. Defect Clustering in GaN Irradiated with $\mathrm{O}^{+}$Ions, J. Mater. Res. 2002, 17, 2945-2952.

(22) Yan, Y.; Dalpian, G. M.; Al-Jassim, M. M.; Wei, S.-H. Energetics and Electronic Structure of Stacking Faults in ZnO. Phys. Rev. B 2004, 70, 193206. 
(23) Blank, H.; Delavignette, P.; Gevers, R.; Amelinckx, S. Fault Structures in Wurtzite. Phys. Status Solidi B 1964, 7, 747 764.

(24) Knoll, S. M.; Rhode, S. K.; Zhang, S.; Joyce, T. B.; Moram, M. A. Defects in Epitaxial ScGaN: Dislocations, Stacking Faults, and Cubic Inclusions. Appl. Phys. Lett. 2014, 104, 101906.

(25) Kovács, A.; Schaffer, B.; Moreno, M. S.; Jinschek, J. R.; Craven, A. J.; Dietl, T.; Bonanni, A.; Dunin-Borkowski, R. E. Characterization of Fe-N Nanocrystals and Nitrogen-Containing Inclusions in $(\mathrm{Ga}, \mathrm{Fe}) \mathrm{N}$ Thin Films Using Transmission Electron Microscopy. J. Appl. Phys. 2013, 114, 033530.

(26) Nickel, N. H.; Gluba, M. A. Defects in Compound Semiconductors Caused by Molecular Nitrogen. Phys. Rev. Lett. 2009, 103, 145501.

(27) Lambrecht, W. R. L.; Boonchun, A. Identification of a N-Related Shallow Acceptor and Electron Paramagnetic Resonance Center in $\mathrm{ZnO}: \mathrm{N}_{2}{ }^{+}$on the Zn Site. Phys. Rev. B 2013, 87, 195207.

(28) Philipps, J. M.; Stehr, J. E.; Buyanova, I.; Tarun, M. C.; McCluskey, M. D.; Meyer, B. K.; Hofmann, D. M. Recharging Behavior of Nitrogen-Centers in ZnO. J. Appl. Phys. 2014, 116, 063701.

(29) Soudi, A.; Khan, E. H.; Dickinson, J. T.; Gu, Y. Observation of Unintentionally Incorporated Nitrogen-Related Complexes in $\mathrm{ZnO}$ and GaN Nanowires. Nano Lett. 2009, 9(5), 1844-1849.

(30) Fons, P.; Tampo, H.; Kolobov, A. V., Ohkubo, M.; Niki, S.; Tominaga, J.; Carboni, R.; Friedrich, S. Direct Observation of Nitrogen Location in Molecular Beam Epitaxy Grown Nitrogen-Doped ZnO. AIP Conf. Proc. 2007, 882, 381-383.

(31) Gao, J.; Qin, R.; Luo, G.; Lu, J; Leprince-Wang, Y.; Ye, H.; Liao, Z.; Zhao, Q.; Yu, D. First-principles Study of the Formation Mechanisms of Nitrogen Molecule in Annealed ZnO. Phys. Lett. A 2010, 374, 3546-3550.

(32) Hull, D.; Bacon, D.J. Introduction to Dislocations $5^{\text {th }}$ Ed.; Elsevier: Oxford, 2011.

(33) Kaiser, U.; Muller, D.A.; Chuvilin, A.; Pasold, G.; Witthuhn, W. The Formation of Clusters and Nanocrystals in ErImplanted Hexagonal Silicon Carbide. Microsc. Microanal. 2004, 10, 301-310. 